\title{
Shake-Up Processes in Photoluminescence of Two-Dimensional Holes in a High Magnetic Field
}

\author{
A. Wóss $^{a}$, L. Bryja $^{a}$, J. Misiewicz $^{a}$, M. Potemski ${ }^{b}$, \\ D. REUTER ${ }^{c}$ AND A. WIECK ${ }^{c}$ \\ ${ }^{a}$ Institute of Physics, Wrocław University of Technology \\ Wybrzeże Wyspiańskiego 27, 50-370 Wrocław, Poland \\ ${ }^{b}$ Grenoble High Magnetic Field Laboratory, CNRS \\ 38042 Grenoble Cedex 9, France \\ ${ }^{c}$ Lehrstuhl fur Festkörperphysik, Ruhr Universität \\ Universitätstr. 150, 44780 Bochum, Germany
}

Recombination of excitons and positive trions is studied by two-beam photoluminescence of a two-dimensional hole gas in a high magnetic field. The singlet, dark-triplet and bright-triplet states of a free trion are resolved, and their binding energies are determined. Recombination of acceptor-bound trions is also detected, including a low-energy cyclotron replica, corresponding to a hole shake-up process. Identification of all these different transitions was possible by analysis of optical selection rules and the comparison of experimental spectra with realistic numerical calculations.

PACS numbers: 71.35.Cc, 71.35.Ji, 73.21.Fg

\section{Introduction}

Magneto-photoluminescence (PL) is a powerful tool in experimental studies of two-dimensional (2D) systems of carriers in the quantum Hall regime [1]. Field evolution of the PL spectrum reveals information about single-particle energies and the exchange interaction with the free carriers.

The bound states that usually dominate the spectra at sufficiently low density and high magnetic field are neutral excitons $(\mathrm{X}=\mathrm{e}+\mathrm{h})$ and trions $\left(\mathrm{X}^{+}=\mathrm{X}+\mathrm{h}\right.$ for the case of the gas of valence holes) $[2,3]$. In analogy to $\mathrm{X}^{-}$states, the trions involving (only) heavy holes are distinguished by pair (pseudo)spin $S=0$ or 1 and angular momentum $M$. The most stable trions are the singlet $\mathrm{X}_{\mathrm{s}}^{+}(S=M=0)$ 
and a pair of triplets $\mathrm{X}_{\mathrm{tb}}^{+}$and $\mathrm{X}_{\mathrm{td}}^{+}(S=1$ and $M=0$ and 1 for the "bright" and "dark" state, respectively) [4].

The main focus of this paper is the shake-up process in which the e-h pair radiative recombination $(\mathrm{e}+\mathrm{h} \rightarrow \gamma)$ is accompanied by a (cyclotron) excitation of another hole to a higher Landau level (LL), $\mathrm{h} \rightarrow \mathrm{h}^{*}[5]$. Clearly, such process involves at least two holes, i.e., a trion. Moreover, angular momentum conservation in photon emission [6] forbids shake-up recombination of free trions, requiring an additional collision to break the 2D translational symmetry [7]. Remarkably, free holes are not very efficient at relaxing the selection rule in neither exciton $\left(\mathrm{X}+\mathrm{h} \rightarrow \gamma+\mathrm{h}^{*}\right)$ nor trion $\left(\mathrm{X}^{+}+\mathrm{h} \rightarrow \gamma+\mathrm{h}+\mathrm{h}^{*}\right)$ scattered emission. Therefore, by requiring interaction with impurities, observation of shake-up transitions allowed us to probe disorder effects in PL (even at a high carrier mobility).

In this paper, we report polarization-resolved PL measurements of a $2 \mathrm{D}$ hole gas at low temperatures $T \geq 1.8 \mathrm{~K}$ and in high magnetic fields $B \leq 23 \mathrm{~T}$. The experiment is supplemented with realistic numerical calculation taking into account quantum well width, LL and subband mixing, etc. The role of neutral acceptors $\mathrm{A}=\mathrm{A}^{-}+\mathrm{h}$ located inside the well in shake-up recombination is understood by the identification of the (weakly) bound $\mathrm{AX}_{\mathrm{d}}^{+}=\mathrm{A}^{-}+\mathrm{e}+3 \mathrm{~h}$ doublet $(S=1 / 2)$ ground state and its emission spectrum into different $\mathrm{A}^{+}=\mathrm{A}^{-}+2 \mathrm{~h}$ states.

\section{Experiment}

The studied sample was a GaAs $/ \mathrm{Ga}_{0.65} \mathrm{Al}_{0.35} \mathrm{Al}$ quantum well of width $w=$ $15 \mathrm{~nm}, \mathrm{MBE}$ grown on a (001) semi-insulating GaAs substrate, $\delta$ C-doped in the barrier on both sides. The sheet concentration and mobility of the holes at $T=4.2 \mathrm{~K}$ were $p=1.51 \times 10^{11} \mathrm{~cm}^{-2}$ and $\mu=1.01 \times 10^{6} \mathrm{~cm}^{2} /(\mathrm{V} \mathrm{s})$.

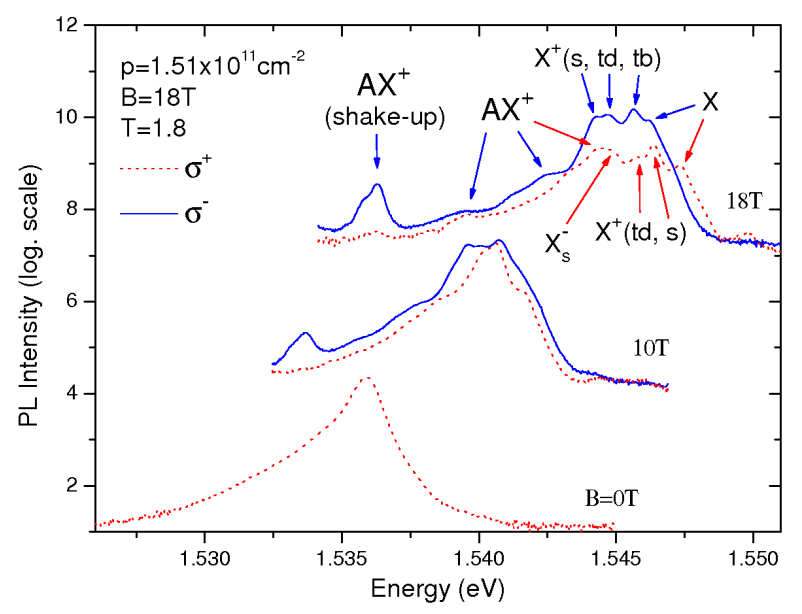

Fig. 1. Polarized PL spectra of a $2 \mathrm{D}$ valence holes in a $15 \mathrm{~nm} \mathrm{GaAs} / \mathrm{Ga}_{0.65} \mathrm{Al}_{0.35} \mathrm{Al}$ quantum well at $B=0,10$, and $18 \mathrm{~T}$ (the three pairs of curves are vertically shifted). Let us note the logarithmic scale on vertical axis. 


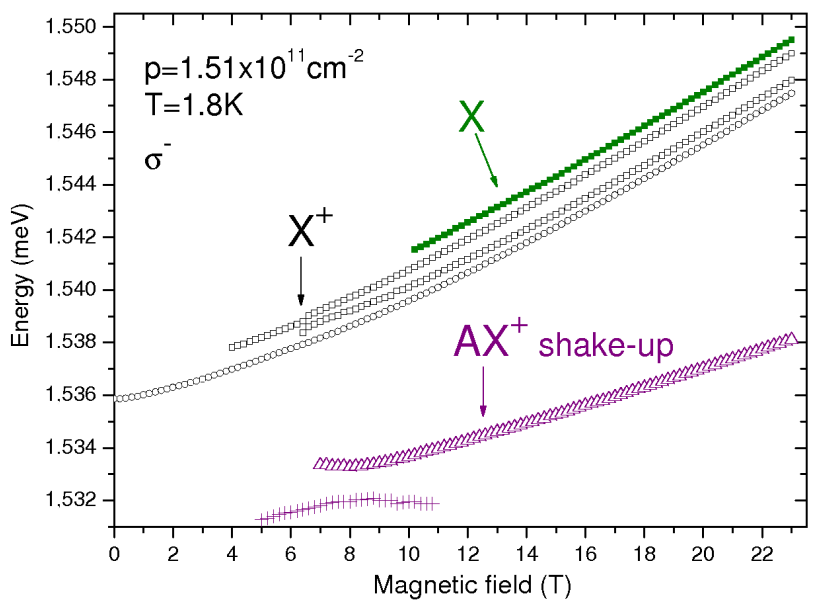

Fig. 2. Field evolution of free exciton and trion recombination, and of the shake-up recombination of the acceptor-bound trion - from the $\sigma^{-}$spectra similar to Fig. 1.

The PL spectra were recorded in the Faraday configuration, with a small field step $\Delta B=0.1 \mathrm{~T}$. To switch between $\sigma^{-}$and $\sigma^{+}$light polarizations, the field direction was changed. PL was excited by the $750 \mathrm{~nm}$ line of titanium sapphire tuneable laser, and an additional ion argon line $514 \mathrm{~nm}$ was used to increase the $2 \mathrm{D}$ electron concentration.

The sample $\sigma^{ \pm}$PL spectra are shown in Fig. 1. Especially interesting is data for $B=18 \mathrm{~T}$, showing all detected emission lines in one spectrum. The individual peaks labeled by arrows were identified by comparison with numerics and by tracing field evolution (shown in Fig. 2 for some states).

\section{Calculation}

The numerical calculations consisted of exact diagonalization of model Hamiltonians of various small $\mathrm{e}-\mathrm{h}$ systems, with and without an additional acceptor (point charge) $\mathrm{A}^{-}$located in the middle of the quantum well. To close boundary conditions, we use the Haldane geometry [8] and map the in-plane motion onto the sphere of radius $R$ with the Dirac monopole of strength $2 Q$ placed in the centre. $2 Q \phi_{0}=4 \pi R^{2} B$ where $\phi_{0}=h c / e$ is flux quantum, and $Q \ell^{2}=R^{2}$ where $\ell=\sqrt{\hbar c / e B}$ is the magnetic length; in the following we show data for $2 Q=20$. The results for free $\mathrm{X}$ and $\mathrm{X}^{+}$'s are similar to those published earlier [4], so here we focus on the $\mathrm{AX}^{+}$.

To explain the $\mathrm{AX}^{+}$energy spectrum let us begin with an ideal case $(w=0$, lowest LL, exact particle-hole e-h symmetry), shown in Fig. 3. The ground state occurs at $L=2 Q=20$, i.e., at $M=0$ (the angular momentum on a sphere $L$ is related to that on a plane $M$ by $L=2 Q-M)$. It is a "multiplicative" state, which means that due to the "hidden symmetry" [9] it has the same energy as an unbound $\mathrm{A}_{\mathrm{s}}^{+}+\mathrm{X}$ configuration. The compact $M=1$ spin-doublet $\mathrm{AX}_{\mathrm{d}}^{+}$ 


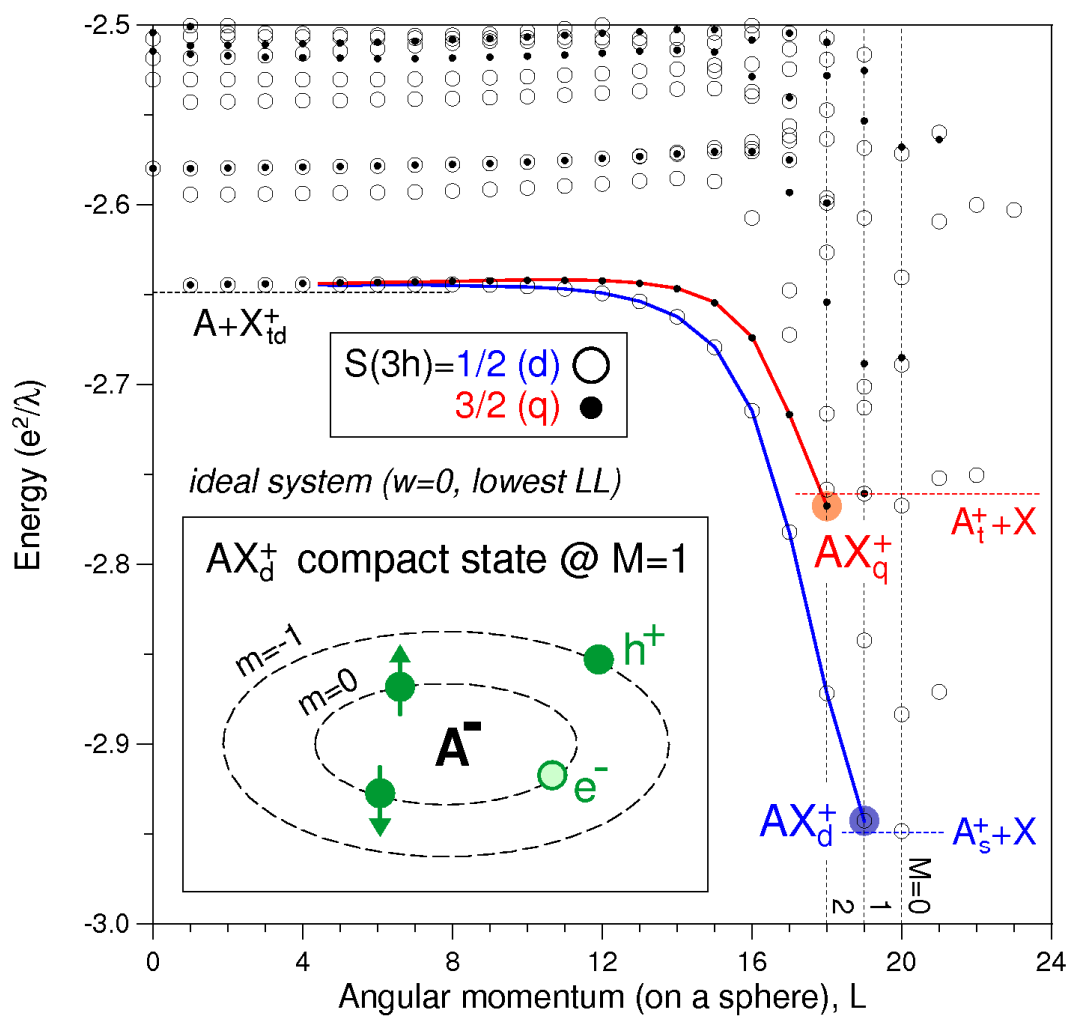

Fig. 3. Energy spectrum of a $3 \mathrm{~h}+$ e system in the presence of an acceptor $\mathrm{A}^{-}$in the plane of the well, calculated on a sphere for the magnetic monopole strength $2 Q=20$. $\lambda$ is the magnetic length. Parameters adequate for an ideal system: $w=0$ and lowest LL. Inset: "compact" $\mathrm{AX}_{\mathrm{d}}^{+}$state with angular momentum $M=1$.

(see inset) marked at $L=19$ is clearly unbound. The spin-polarized quadruplet $\mathrm{AX}_{\mathrm{q}}^{+}$is also marked. It does not break up into $\mathrm{A}_{\mathrm{t}}^{+}+\mathrm{X}$, but its stability would require unrealistically high Zeeman energy. The solid lines connect series of states originating from $\mathrm{AX}^{+}$at small $M$ (high $L$ ) and going toward higher $M$ 's, and describing an $\mathrm{X}^{+}$orbiting around an $\mathrm{A}$. Thus, they measure the $\mathrm{A}-\mathrm{X}^{+}$interaction pseudopotential, with $M$ playing role of the relative angular momentum (let us note that $\mathrm{X}_{\mathrm{td}}^{+}$is the only bound trion in the lowest LL with exact particle-hole e-h symmetry).

A more realistic $\mathrm{AX}^{+}$spectrum is shown in Fig. 4. It was obtained by using the correct (for $w=15 \mathrm{~nm}$ ) lowest-subband wave functions in the normal direction, and by including two LLs $(n \leq 1)$ for both e and h, with the cyclotron gaps and the magnetic length appropriate for $B=15 \mathrm{~T}$. The main result is the (weak) binding of a pair of $\mathrm{AX}_{\mathrm{d}}^{+}$states at $M=0$ and 1 (i.e., stability against the $\mathrm{A}_{\mathrm{s}}^{+}+\mathrm{X}$ break-up). Binding of the "compact" $M=1$ state is enhanced by 


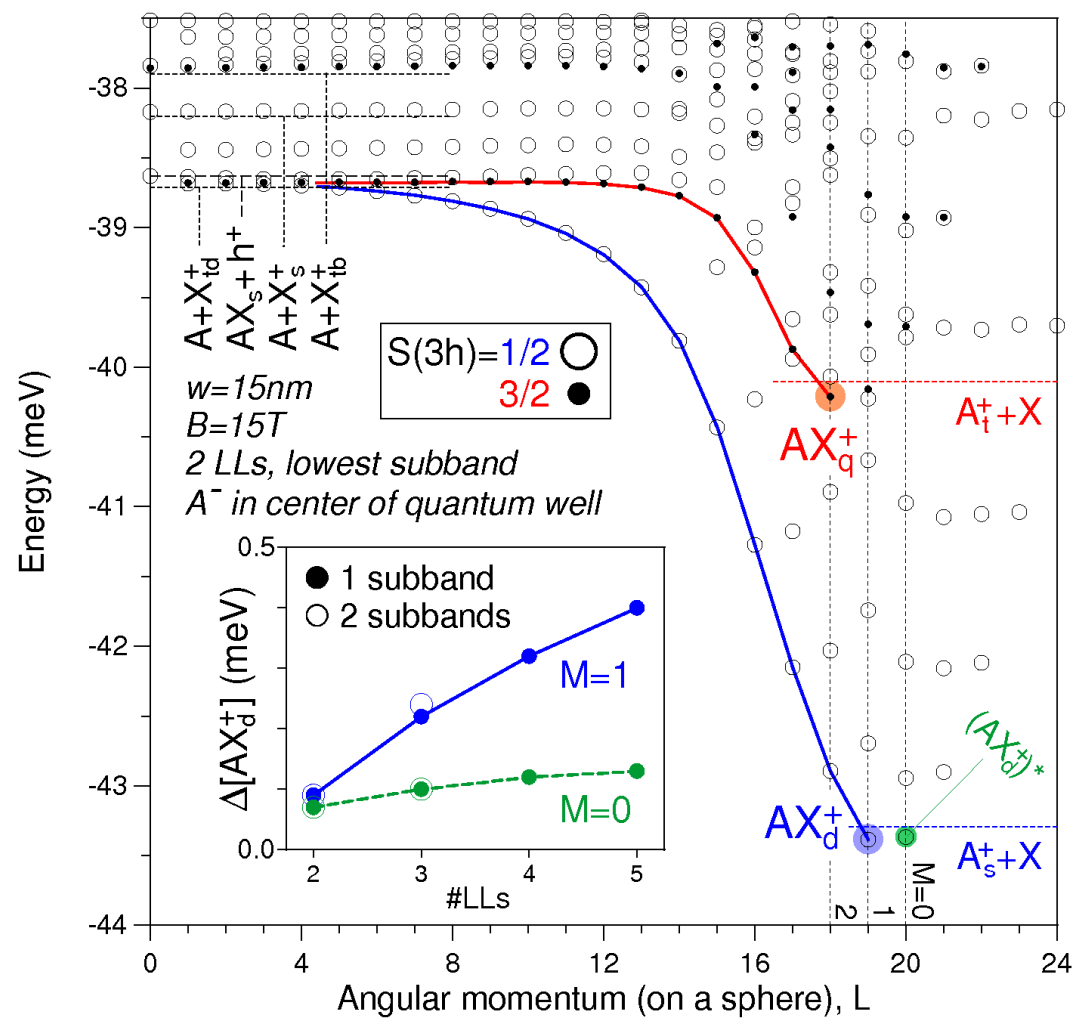

Fig. 4. Similar to Fig. 3 but for $w=15 \mathrm{~nm}$ and $B=15 \mathrm{~T}$, including one subband and two LLs. Inset: binding energies obtained including more subbands and LLs.

inclusion of even more LLs and higher quantum well subbands (see inset). While it is difficult to estimate reliably its binding energy $\Delta$, we conclude that (i) this is the lowest-energy state for an excitonic complex in the presence of acceptors inside the well, and (ii) it is rather weakly bound and requires low temperatures to remain stable (both conclusions agree with our experiment).

Let us now turn to the energy spectrum of the final state in the $\mathrm{AX}_{\mathrm{d}}^{+} \rightarrow$ $\mathrm{A}^{+}+\gamma$ recombination. The result of calculation including as many as five LLs and three subbands for each e and $\mathrm{h}$ is shown in Fig. 5. The $\Delta M=0$ selection rule selects $M=1$ as the only active optical channel (excluding e.g. the singlet ground state at $M=0$ ). Four lowest $M=1$ states have been marked, corresponding to one singlet and one doublet for each following LL configuration: with both holes in the lowest LL: $\mathrm{A}^{+}$, or with one of the holes excited to the $n=1 \mathrm{LL}:\left(\mathrm{A}^{+}\right)^{*}$. The binding energy $\Delta$ of each of these states is measured from an unbound $\mathrm{A}+\mathrm{h}$ or $\mathrm{A}+\mathrm{h}^{*}$ state $\left(\mathrm{h}^{*}\right.$ denotes a hole in the excited $\mathrm{LL}$ ), and indicated in the units of meV. Both triplets are bound with essentially the same binding energy $\left(\Delta_{\mathrm{t}}=\Delta_{\mathrm{t}}^{*}=1.35 \mathrm{meV}\right)$, while both singlets appear to be unbound $\left(\Delta_{\mathrm{s}}=\Delta_{\mathrm{s}}^{*}=0\right)$. 


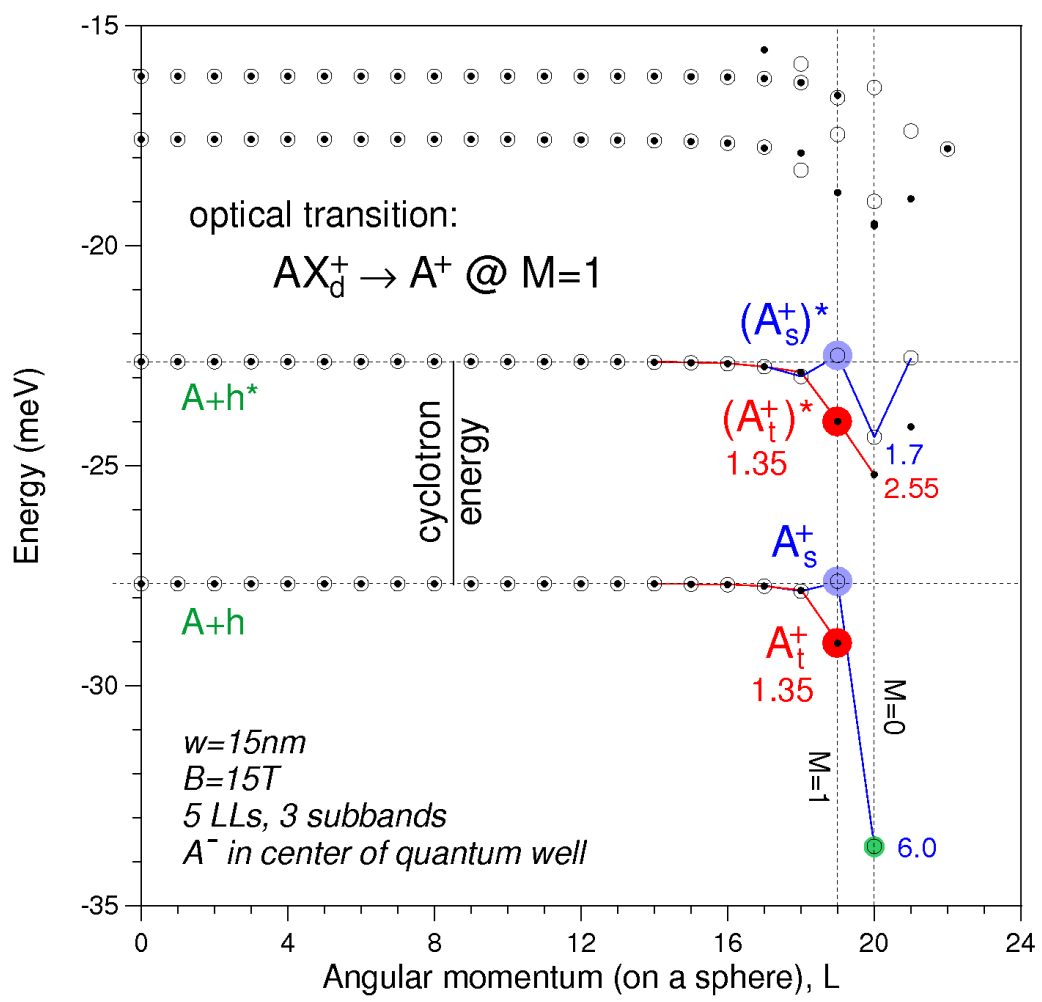

Fig. 5. Energy spectrum of $2 \mathrm{~h}$ with an acceptor $\mathrm{A}^{-}$in the middle of quantum well, calculated for $w=15 \mathrm{~nm}$ and $B=15 \mathrm{~T}$, including three subbands and five LLs.

Four different final states yield (up to) four distinct lines in the PL spectrum. The evaluated optical oscillator strengths (relative to $\mathrm{X}$ ) are: $I_{\mathrm{t}}=0.0061$, $I_{\mathrm{s}}=0.0012, I_{\mathrm{t}}^{*}=0.0008$, and $I_{\mathrm{s}}^{*} \approx 0$, in good agreement with our experiment. Remarkably, since $\Delta_{\mathrm{t}}=\Delta_{\mathrm{t}}^{*}$, the shake-up $\mathrm{AX}_{\mathrm{d}}^{+} \rightarrow\left(\mathrm{A}_{\mathrm{t}}^{+}\right)^{*}$ transition occurs at exactly the (hole) cyclotron energy below its $\mathrm{AX}_{\mathrm{d}}^{+} \rightarrow \mathrm{A}_{\mathrm{t}}^{+}$parent transition. Generally, the lack of the Coulomb contribution to this peak splitting allows for extraction of the hole cyclotron mass from PL.

\section{Conclusion}

In conclusion, by combining polarization-resolved PL with realistic numerical calculations we have detected and explained shake-up recombination of acceptor-bound trions in a $2 \mathrm{D}$ valence hole gas in a high magnetic field. The position of the shake-up line allows for the estimate of a hole cyclotron mass from PL. We also found that, in the absence of impurities, shake-up of free excitons and trions scattered by free holes is negligible. 


\section{Acknowledgments}

The authors greatly acknowledge support from grants: RITA-CT-2003505474 of EC and N20210431/0771 of the MENiS (Poland).

\section{References}

[1] I.V. Kukushkin, V.B. Timofeev, Adv. Phys. 45, 147 (1996).

[2] M.A. Lampert, Phys. Rev. Lett. 1, 450 (1958).

[3] K. Kheng, R.T. Cox, M.Y. d'Aubigné, F. Bassani, K. Saminadayar, S. Tatarenko, Phys. Rev. Lett. 71, 1752 (1993); H. Buhmann, L. Mansouri, J. Wang, P.H. Beton, N. Mori, L. Eaves, M. Henini, M. Potemski, Phys. Rev. B 51, 7969 (1995); G. Yusa, H. Shtrikman, I. Bar-Joseph, Phys. Rev. Lett. 87, 216402 (2001); D. Andronikov, V. Kochereshko, A. Platonov, T. Barrick, S.A. Crooker, G. Karczewski, Phys. Rev. B 72, 165339 (2005).

[4] B. Stebe, A. Ainane, Superlatt. Microstruct. 5, 545 (1989); A. Wójs, P. Hawrylak, Phys. Rev. B 51, 10880 (1995); D.M. Whittaker, A.J. Shields, ibid. 56, 15185 (1997); A. Wójs, J.J. Quinn, P. Hawrylak, ibid. 62, 4630 (2000); C. Riva, F.M. Peeters, K. Varga, ibid. 63, 115302 (2001).

[5] G. Finkelstein, H. Shtrikman, I. Bar-Joseph, Phys. Rev. B 53, 12593 (1996); ibid. 56, 10326 (1997); S. Glasberg, H. Shtrikman, I. Bar-Joseph, ibid. 63, 201308 (2001).

[6] J.J. Palacios, D. Yoshioka, A.H. MacDonald, Phys. Rev. B 54, 2296 (1996); A.B. Dzyubenko, A.Y. Sivachenko, Phys. Rev. Lett. 84, 4429 (2000).

[7] A.B. Dzyubenko, Phys. Rev. B 69, 115332 (2004); also in Proc. 28th Int. Conf. on the Physics of Semiconductors ICPS-2006, Vienna, (Austria) 2006, AIP Conf. Proc., to be published.

[8] F.D.M. Haldane, Phys. Rev. Lett. 51, 605 (1983).

[9] A.B. Dzyubenko, Yu.E. Lozovik, Fiz. Tverd. Tela 25, 1519 (1983); A.H. MacDonald, E.H. Rezayi, Phys. Rev. B 42, 3224 (1990). 